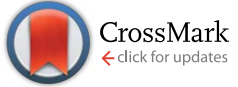

Cite this: RSC Adv., 2014, 4, 61580

Received 12th September 2014 Accepted 4th November 2014

DOI: $10.1039 / \mathrm{c} 4 \mathrm{ra10315g}$

www.rsc.org/advances

\title{
Glycogen-graft-poly(2-alkyl-2-oxazolines) - the new versatile biopolymer-based thermoresponsive macromolecular toolbox
}

\author{
Aneta Pospisilova, ${ }^{a}$ Sergey K. Filippov, ${ }^{a}$ Anna Bogomolova, ${ }^{a}$ Stuart Turner, ${ }^{b}$ \\ Ondrej Sedlacek, ${ }^{a}$ Nikolai Matushkin, ${ }^{a}$ Zulfia Cernochova, ${ }^{a}$ Petr Stepanek ${ }^{a}$ \\ and Martin Hruby ${ }^{\star a}$
}

This study is focused on thermoresponsive glycogen-graft-poly(2-alkyl-2-oxazolines), a new group of nanostructured hybrid dendrimeric stimuli-responsive polymers connecting the body's own biodegradable polysaccharidic dendrimer glycogen with the widely tuneable thermoresponsive behavior of polypeptide-analogic poly(2-alkyl-2-oxazolines), which are known to be biocompatible. Glycogengraft-poly(2-alkyl-2-oxazolines) were prepared by a simple one-pot two-step procedure involving cationic ring-opening polymerization of 2-alkyl-2-oxazolines followed by termination of the living cationic ends with sodium glycogenate. As confirmed by light and X-ray scattering, as well as cryotransmission electron microscopy, the grafted dendrimer structure allows easy adjustment of the cloud point temperature, the concentration dependence and nanostructure of the self-assembled phase separated polymer by crosstalk during graft composition, the graft length and the grafting density, in a very wide range.

\section{Introduction}

Glycogen (GG) is the main storage polysaccharide in animals, and is mainly contained in the liver ${ }^{1}$ and muscles. ${ }^{2}$ The average content in a human is around $100 \mathrm{~g}^{3}{ }^{3}$ Glycogen is a hyperbranched poly (D-glucose ${ }^{1}$ ) with $\mathrm{D}$-glucose units connected by $\alpha(1 \rightarrow 4)$ bonds, and branching occurring via $\alpha(1 \rightarrow 6)$ bonds. ${ }^{4}$ It is a natural biodegradable dendrimer with a small protein core (glycogenin). It has a spherical shape with a diameter of $c a$. $50 \mathrm{~nm}$, a narrow size distribution and a weight-average molecular weight $\left(M_{\mathrm{w}}\right)$ of several MDa depending on the source organism. Biosynthesis and degradation of glycogen in humans proceeds under physiological conditions only intracellularly and the glycogen is almost inert to amylases present in the bloodstream. ${ }^{1,5}$ This means that glycogen should only be very slowly degraded after intravenous (i.v.) administration to the bloodstream (its molecular weight is well above the renal threshold, so it cannot be directly eliminated by the kidneys without previous biodegradation), but should be rapidly degraded to D-glucose after internalization by cells. Due to these properties, it is a very promising construction nanomaterial for biomedical materials. ${ }^{6}$

\footnotetext{
${ }^{a}$ Institute of Macromolecular Chemistry, Academy of Sciences of the Czech Republic, $v$. v. i., Heyrovsky Sq. 2, 16206 Prague 6, Czech Republic. E-mail: mhruby@centrum.cz; Fax: +420 296809 410; Tel: +420296809230

${ }^{b}$ EMAT, University of Antwerp, B-2020 Antwerp, Belgium
}

Highly defined or so-called precision polymers have been a hot topic in recent years and are particularly interesting for biomedical applications. ${ }^{7-9}$ Poly(2-alkyl-2-oxazolines) (POXs) are a group of such biocompatible synthetic polymers with a polypeptide-isomeric structure. They are prepared by living cationic ring-opening polymerization, ${ }^{7-9}$ which provides excellent control over the chain length, molar mass distribution and chain-end functionality and enables preparation of well-defined block copolymers. Thanks to the versatility of the monomers and the advantageous character of cationic polymerization, the preparation of polymers with various architectures is possible and enables easy tuning of the polymer properties. POXs may be tailored to be fully hydrophilic, thermoresponsive (with a lower critical solution temperature, LCST),${ }^{10}$ hydrophobic or fluorophilic, depending on the alkyl moiety used. Due to the living nature of the polymerization the chain length may be precisely adjusted and an appropriate choice of monomer(s), initiator and termination agent enables us to functionalize the polymer at any position in a defined way. ${ }^{11}$

Hybrid copolymers comprising natural and synthetic building blocks are becoming an increasingly important class of functional materials. ${ }^{12-14}$ They combine the sustainable environment-friendly nature, biodegradability and structure of natural polymers with the processability, tailorability and economy of synthetic polymers. These materials are homogeneous on a macroscopic scale but can be designed to undergo micro- or nanophase separation/self-assembly, which generates 
materials that can be useful as, inter alia, delivery systems for bioactive species or tissue engineering scaffolds. ${ }^{15-17}$

In this paper we describe a completely new concept of biocompatible hybrid copolymers based on glycogen-graftpoly(2-alkyl-2-oxazolines). Both glycogen and POXs are biocompatible and therefore their hybrid is expected to be biocompatible. The POXs were polymerized in a living manner with the initiating alkylating agent able to choose one end of the monomer and the molecular weight adjustable by altering the initiator: monomer ratio. The living cationic end after this polymerization was used to alkylate the alcoholate of glycogen, forming the glycogen-graft-poly(2-alkyl-2-oxazolines). This easy two-step one-pot synthesis creates a conceptually new, versatile, nanostructured platform of hybrid sphere-shaped biodegradable polysaccharide dendrimer-based copolymers, with a thermoresponsive behavior that is adjustable over a wide range by variation of the POXs chain length, grafting density and reactive chain ends. Special focus is therefore given to the in vitro temperature-dependent behavior of these materials in solution. Such thermoresponsive polymers are potentially suitable as drug releasing depots, self-organized nanostructured tissue engineering scaffolds or materials for injectable brachytherapy, where widely adjustable properties, temperature-responsivity, biodegradability and biocompatibility are advantageous. ${ }^{15,18}$

\section{Materials and methods}

\section{Materials}

2-Butyl-2-oxazoline and 2-isopropyl-2-oxazoline were prepared according to ref. 19. Diethyl ether and sodium chloride were purchased from Lachner Ltd. (Neratovice, Czech Republic), all other chemicals were purchased from Sigma-Aldrich Ltd. (Prague, Czech Republic). The chemicals were used as provided unless stated otherwise.

\section{Methods}

Preparation of glycogen-graft-poly-(2-isopropyl-2-oxazoline), theoretical molecular weight of POX grafts $M_{\mathrm{n}, \text { graft }} \approx 500 \mathrm{Da}$ (see Table 1). The starting mixture contained 2-isopropyl-2oxazoline $(3.333 \mathrm{~mL}, 28.0 \mathrm{mmol})$ and allyl bromide $(0.595 \mathrm{~mL}$, $6.9 \mathrm{mmol}$ ). The starting mixture was mixed with anhydrous acetonitrile $(5 \mathrm{~mL})$ and the solution was polymerized overnight at $70{ }^{\circ} \mathrm{C}$ under an argon atmosphere.

Glycogen (type II from oysters, $3.5 \mathrm{~g}$ ) was dissolved in anhydrous dimethyl sulfoxide $(50.5 \mathrm{~mL})$ and the solution was azeotropically dried using the addition $(10 \mathrm{~mL})$ and subsequent evaporation of anhydrous toluene. Sodium hydride $(1.4 \mathrm{~g}$ of a $60 \%$ dispersion in mineral oil, $35 \mathrm{mmol}$ ) was added to the glycogen solution and the mixture was stirred at $70{ }^{\circ} \mathrm{C}$ until hydrogen gas evolved (ca. $2 \mathrm{~h}$ ). The glycogen containing solution was split into dry flasks [30 g (GG-PIPO1, GG-PIPO21 and GGPIBO1), $20 \mathrm{~g}$ (GG-PIPO2, GG-PIPO22 and GG-PIBO2) or $10 \mathrm{~g}$ (GG-PIPO3, GG-PIPO23 and GG-PIBO3), respectively] and the polyoxazoline polymerization mixture was added $[1.17 \mathrm{~g}$ (GGPIPO1, GG-PIPO21 and GG-PIBO1), $2.33 \mathrm{~g}$ (GG-PIPO2, GGPIPO22 and GG-PIBO2) or $3.50 \mathrm{~g}$ (GG-PIPO3, GG-PIPO23 and
GG-PIBO3), respectively]. Each resulting mixture was stirred for $3 \mathrm{~h}$ at $70{ }^{\circ} \mathrm{C}$. Water $(5 \mathrm{~mL})$ was added, the solution was twice washed of the mineral oil using diethyl ether and then the aqueous layer was dialyzed against water for $72 \mathrm{~h}$ (molecular weight cut-off 6800, to remove unbound POX and other components from the reaction mixture) and freeze dried.

According to this procedure, we prepared three groups of glycogen-graft-polyoxazolines with different polyoxazoline graft properties, with three polymers in each group that varied in their theoretical content of polyoxazoline.

Yields: $670 \mathrm{mg}$ (29\%) of GG-PIPO1, $640 \mathrm{mg}$ (27\%) of GGPIPO2 and $440 \mathrm{mg}$ (19\%) of GG-PIPO3. N: 1.09\% (GG-PIPO1), $2.91 \%$ (GG-PIPO2) and 4.64\% (GG-PIPO3). ${ }^{1} \mathrm{H}-\mathrm{NMR}(300 \mathrm{MHz}$, $\left.\mathrm{D}_{2} \mathrm{O}\right), \delta(\mathrm{ppm}): 5.9\left(\mathrm{~m}, \mathrm{POX}: \mathrm{CH}_{2}=\mathrm{CH}-\right), 5.4$ (s, glucose: $\left.\mathrm{H} 1\right)$, 5.3-5.0 (m, POX: $\mathrm{CH}_{2}=\mathrm{CH}-$ ) , 4.1-3.5 (m, glucose: H2-H6, POX: $-\mathrm{CH}_{2}-$ ), 2.9-2.7 (d, POX: - $\left.\mathrm{CH}-\left(\mathrm{CH}_{3}\right)_{2}\right), 1.1$ (d, POX: - $\left.\mathrm{CH}-\left(\mathrm{CH}_{3}\right)_{2}\right)$.

The content of polyoxazoline $w_{\mathrm{ox}}(\mathrm{m} / \mathrm{m})$ was calculated according to eqn (1):

$$
w_{\mathrm{ox}}=\frac{w_{\mathrm{N}}}{w_{\mathrm{N}, \mathrm{ox}}}
$$

where $w_{\mathrm{N}}$ is the content of nitrogen in the sample and $w_{\mathrm{N}, \mathrm{ox}}$ is the calculated content of nitrogen in the polyoxazoline graft.

The content of polyoxazoline was found to be $10 \%$ (GGPIPO1), 26\% (GG-PIPO2) and 41\% (GG-PIPO3).

Preparation of glycogen-graft-poly-(2-isopropyl-2-oxazoline), $\boldsymbol{M}_{\mathbf{n}, \text { theoretical }}($ POX) $\approx \mathbf{5 0 0 0}$ Da. See the Preparation of glycogen-graft-poly-(2-isopropyl-2-oxazoline), theoretical molecular weight of POX grafts $M_{\mathrm{n} \text {,graft }} \approx 500$ Da for the general procedure. Here, the starting mixture contained 2-isopropyl-2oxazoline $(4.110 \mathrm{~mL}, 34.6 \mathrm{mmol})$ and allyl bromide $(68.1 \mu \mathrm{L}$, $0.8 \mathrm{mmol})$.

Yields: $1230 \mathrm{mg}$ (53\%) of GG-PIPO21, $1110 \mathrm{mg}$ (48\%) of GGPIPO22 and $1270 \mathrm{mg}$ (55\%) of GG-PIPO23. N: $3.18 \%$ (GGPIPO21), 5.39\% (GG-PIPO22) and 7.96\% (GG-PIPO23). ${ }^{1} \mathrm{H}-$ NMR (300 MHz, $\mathrm{D}_{2} \mathrm{O}$ ), $\delta$ (ppm): 5.4 (s, glucose: H1), 4.1-3.5 (m, glucose: H2-H6, POX: - $\mathrm{CH}_{2}-$ ), 2.9-2.7 (d, POX: - $\left.\mathrm{CH}-\left(\mathrm{CH}_{3}\right)_{2}\right)$, 1.1 (d, POX: - $\left.\mathrm{CH}-\left(\mathrm{CH}_{3}\right)_{2}\right)$.

The content of polyoxazoline $(\mathrm{m} / \mathrm{m})$ was calculated according to eqn (1) and was found to be $25 \%$ (GG-PIPO21), 44\% (GGPIPO22) and 64\% (GG-PIPO23).

Preparation of glycogen-graft-poly-(2-isopropyl-2-oxazolineco-2-butyl-2-oxazoline), $M_{\mathrm{n} \text {,theoretical }}(\mathrm{POX}) \approx 2500$ Da. See the Preparation of glycogen-graft-poly-(2-isopropyl-2-oxazoline), theoretical molecular weight of POX grafts $M_{\mathrm{n}, \text { graft }} \approx 500 \mathrm{Da}$ for the general procedure. Here, the starting mixture contained 2-isopropyl-2-oxazoline (3.111 $\mathrm{mL}, 27.5 \mathrm{mmol})$, 2-butyl-2oxazoline (0.794 g, $6.3 \mathrm{mmol})$ and allyl bromide $(124 \mu \mathrm{L}, 1.6$ mmol).

Yields: $1700 \mathrm{mg}$ (73\%) of GG-PIBO1, $900 \mathrm{mg}$ (39\%) of GGPIBO2 and $600 \mathrm{mg}$ (26\%) of GG-PIBO3. N: 0.71\% (GG-PIBO1), $3.52 \%$ (GG-PIBO2) and 5.32\% (GG-PIBO3). ${ }^{1} \mathrm{H}-\mathrm{NMR}(300 \mathrm{MHz}$, $\left.\mathrm{D}_{2} \mathrm{O}\right), \delta(\mathrm{ppm}): 5.4$ (s, glucose: $\left.\mathrm{H} 1\right), 4.1-3.5$ (m, glucose: $\mathrm{H} 2-\mathrm{H} 6$, POX: $-\mathrm{CH}_{2}-$ ), 2.9-2.7 (d, POX: $\left.-\mathrm{CH}-\left(\mathrm{CH}_{3}\right)_{2}\right), 2.4$ (t, POX: $-\mathrm{CH}_{2}-$ $\mathrm{CH}_{2}-\mathrm{CH}_{2}-\mathrm{CH}_{3}$ ), 1.5 (m, POX: $-\mathrm{CH}_{2}-\mathrm{CH}_{2}-\mathrm{CH}_{2}-\mathrm{CH}_{3}$ ), 1.3 (m, POX: $-\mathrm{CH}_{2}-\mathrm{CH}_{2}-\mathrm{CH}_{2}-\mathrm{CH}_{3}$ ), 1.1 (d, POX: $\left.-\mathrm{CH}-\left(\mathrm{CH}_{3}\right)_{2}\right), 0.9$ (t, POX: $\left.-\mathrm{CH}_{2}-\mathrm{CH}_{2}-\mathrm{CH}_{2}-\mathrm{CH}_{3}\right)$. 
The content of polyoxazoline $(\mathrm{m} / \mathrm{m})$ was calculated according to eqn (1) and was found to be 6\% (GG-PIBO1), 29\% (GG-PIBO2) and $44 \%$ (GG-PIBO3).

The number-average $\left(M_{\mathrm{n}}\right)$ molecular weight of the graft was determined by gel permeation chromatography ${ }^{20}$ of the polymerization mixture before the addition to glycogen and was found to be $428 \mathrm{Da}$ for poly-(2-isopropyl-2-oxazoline) with $M_{\mathrm{n} \text {,theoretical }}=500 \mathrm{Da}, 5400 \mathrm{Da}$ for poly-(2-isopropyl-2-oxazoline) with $M_{\mathrm{n} \text {,theoretical }}=5000 \mathrm{Da}$ and $2400 \mathrm{Da}$ for poly-(2-isopropyl-2oxazoline-co-2-butyl-2-oxazoline) with $M_{\mathrm{n} \text {,theoretical }}=2500 \mathrm{Da}$, with the polydispersity $I=M_{\mathrm{w}} / M_{\mathrm{n}} \sim 1.2$ in all cases. The absolute molecular weight distribution was calculated from a chromatogram by combination of the refraction index and multiangle light scattering (MALS) detector signals using Wyatt Technology ASTRA® 6 software (Wyatt Technology Co., Santa Barbara, CA, U.S.A.).

Cloud point temperature measurement. The cloud point temperature (CPT) was determined optically as the increase in turbidity (onset temperature of macroscopic phase separation) of the polymer solution of a given concentration in physiological saline solution.

Dynamic light scattering (DLS). The particle size distribution in solution was measured on an ALV instrument equipped with a $22 \mathrm{~mW} \mathrm{He}-\mathrm{Ne}$ laser at an angle of $90^{\circ}$. The correlation functions were analyzed by REPES, with analytical software ${ }^{21}$ providing the distribution function of the hydrodynamic radii, $G\left(R_{\mathrm{h}}\right)$. To account for the logarithmic scale on the $R_{\mathrm{h}}$ axis, all DLS distribution diagrams are shown in the equal area representation, ${ }^{22} R_{\mathrm{h}} G\left(R_{\mathrm{h}}\right)$. In all experiments, about $2 \mathrm{~mL}(c=1.0$ $\mathrm{wt} \%$ ) of the sample solution was filtered using a $0.22 \mu \mathrm{m}$ PVDF filter and transferred to a sealed dust-free light-scattering cell. The temperature was controlled within $0.05{ }^{\circ} \mathrm{C}$. The hydrodynamic radius of the nanoparticles, $R_{\mathrm{h}}$, was calculated using the Stokes-Einstein equation.

Cloud point temperatures (CPTs) for each particular concentration in water, in addition to the CPT in physiological saline solution, were determined from the temperature dependences of the particle hydrodynamic radius, $R_{\mathrm{h}}$, and the scattering intensity, $I_{\mathrm{s}}$, that were measured at the scattering angle $\theta=173^{\circ}$ on a Zetasizer Nano-ZS, Model ZEN3600 (Malvern Instruments, UK). The DTS (Nano) program was used for data evaluation.

Small angle X-ray scattering (SAXS). Synchrotron SAXS experiments were performed on the EMBL beam line P12 (Petra III, Hamburg, Germany) using a pixel detector (2M PILATUS). The X-ray scattering images were recorded for a sampledetector distance of $3.1 \mathrm{~m}$, using a monochromatic incident $\mathrm{X}$ ray beam $(\lambda=0.125 \mathrm{~nm})$ covering the range of momentum transfer $0.05 \mathrm{~nm}^{-1}<q<4.5 \mathrm{~nm}^{-1}(q=4 \pi \sin \theta / \lambda$, where $2 \theta$ is the scattering angle). Most of the samples had no detected measurable radiation damage, by comparison of twenty successive time frames with $50 \mathrm{~ms}$ exposures. In all of the cases reported in this paper, the two-dimensional scattering patterns were isotropic. They were azimuthally averaged to yield the dependence of the scattered intensity $I_{\mathrm{s}}(q)$ on the momentum transfer $q$. Prior to fitting analysis, the solvent scattering was subtracted.
All data manipulations were performed using PRIMUS software. The forward scattering $I_{\mathrm{s}}(q=0)$, and the radius of gyration $R_{\mathrm{g}}$ were evaluated using the Guinier approximation. For further modeling, the data was brought to absolute scale by subtracting an empty cell measurement from a pure water measurement, and scaling by the ratio of the theoretical forward scattering of water and the experimental forward scattering intensity of water.

Isothermal titration calorimetry. Experiments were carried out using the isothermal titration calorimeter MicroCal ITC200 (GE Healthcare, USA). Glycogen solutions $(0.14 \mu \mathrm{M})$ and GGPIPO solutions $(0.1 \mu \mathrm{M})$ in the calorimeter cell were titrated with bovine serum albumin (BSA) solution $(15 \mu \mathrm{M})$ using 20 successive automatic injections of $2 \mu \mathrm{L}$ each. The integration of the peak corresponding to each injection and correction for the baseline were carried out using Origin-based software provided by the manufacturer. A blank experiment, BSA titration of water, was also performed.

Transmission electron microscopy. Transmission electron microscopy (TEM) was performed on a FEI Titan microscope equipped with an aberration corrector and EDAX X-ray spectrometer, operated at $80 \mathrm{kV}$ acceleration voltage. The polymer was stained with uranium salts to increase TEM contrast.

\section{Results and discussion}

All polymers were prepared by the cationic ring opening polymerization of 2-alkyl-2-oxazoline monomers using allyl bromide as the initiator. Using allyl bromide introduces a double bond at the chain end distant from glycogen, which allows further functionalization by, e.g., thiol-click chemistry or radical crosslinking/copolymerization. The living ends were terminated with sodium glycogenate. This procedure is illustrated in Fig. 1.

We successfully synthesized two groups of glycogen-graftpoly(2-isopropyl-2-oxazoline) polymers, which varied in the molar mass of the polyoxazoline graft (500 Da for GG-PIPO1, GG-PIPO2 and GG-PIPO3 and 5000 Da for GG-PIPO21, GGPIPO22, GG-PIPO23, respectively), with three products in each group that varied in their POX content. This allowed us to follow

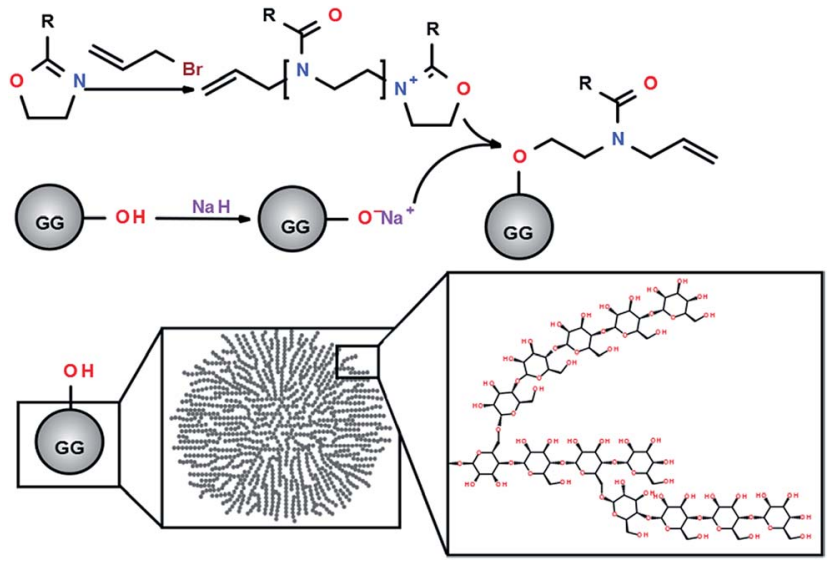

Fig. 1 The structure of natural glycogen and the general synthetic procedure for the glycogen-graft-poly(2-alkyl-2-oxazolines). 
the solution behavior as a function of temperature in the context of both graft length and the weight fraction of thermoresponsive polymer in the hybrid graft copolymer (theoretical content of thermoresponsive polymer $25 \mathrm{wt} \%$ for GGPIPO1, GG-PIPO21 and GG-PIBO1, $50 \mathrm{wt} \%$ for GG-PIPO2, GGPIPO22 and GG-PIBO2 and 75\% for GG-PIPO3, GG-PIPO23 and GG-PIBO3). We characterized the polymers using ${ }^{1} \mathrm{H}$ NMR spectroscopy (confirming the formation of the hybrid), elemental analysis (CHN), TEM, small angle X-ray scattering (SAXS) and dynamic light scattering (DLS). We also prepared three glycogen-graft-poly(2-isopropyl-2-oxazoline-co-2-butyl-2oxazolines) (GG-PIBO1, GG-PIBO2 and GG-PIBO3), where the 2-butyl-2-oxazoline hydrophobic moiety decreases the CPT below body temperature $\left(37^{\circ} \mathrm{C}\right)$, which is essential for future use in biomedical applications. An overview of the prepared polymers is shown in Table 1.

We calculated the obtained content of polyoxazoline using the elemental analysis of nitrogen, because glycogen contains almost no nitrogen (less than $0.1 \mathrm{wt} \%$, glycogenin primer may be neglected) while the POX grafts contain a high amount of nitrogen. The grafts with $M_{\mathrm{n} \text {,theoretical }}=5000$ Da gave POX contents that were only slightly lower than the theoretical value - $25 \mathrm{wt} \%$ for GG-PIPO21, $44 \mathrm{wt} \%$ for GG-PIPO22 and $64 \mathrm{wt} \%$ for GG-PIPO23, the corresponding theoretical values are $25 \%, 50 \%$ and $75 \%$, which shows a high grafting efficiency. In contrast to this, with the lower-molecular-weight grafts of $M_{\mathrm{n} \text {,theoretical }}=$ 500 , the grafting yields were lower $-10 \%$ for GG-PIPO1, $26 \%$ for GG-PIPO2 and 41\% for GG-PIPO3, the corresponding theoretical values are again $25 \%, 50 \%$ and $75 \%$. This apparent paradox that smaller grafts offer lower grafting yields may be explained by the limited sterical accessibility of the D-glucose hydroxyl groups towards alkylation. There is obviously a limit in the number of chains per D-glucose unit; for grafts with $M_{\mathrm{n} \text {,theoretical }}=500$ the value of the POX content of $41 \mathrm{wt} \%$ (GGPIPO3) means one graft per 4.44 D-glucose units, while for grafts with $M_{\mathrm{n} \text {,theoretical }}=5000$ the value of $64 \mathrm{wt} \%$ (GG-PIPO23) means one graft per 17.34 D-glucose units. To exclude the possibility of contamination of the hybrid copolymer with POX grafts unbound to glycogen, the solutions were dialysed using a membrane with a cutoff of $6800 \mathrm{Da}$, which is well above the molecular weight of the polymer grafts.
The limited conformational mobility and therefore also the sterical accessibility of the D-glucose units inside the hyperbranched coil was also confirmed by ${ }^{1} \mathrm{H}$ NMR, where the signals of the polysaccharide part are broad, which is especially obvious for the acetal hydrogen signal at 5.4 ppm (see Fig. 2 for spectra). Unfortunately this broadening makes a quantitative evaluation (integration of the ${ }^{1} \mathrm{H}$ NMR spectra) difficult in our case.

However, the POX grafts are probably not only on the surface of the glycogen unimolecular nanoparticle, as proven by TEM (see Fig. 3). One can clearly see that while below the cloud point temperature (Fig. 3a) the size of the spherical particles is $c a .70$ $\mathrm{nm}$, above the CPT the size of the particles in the aggregates is roughly around $17 \mathrm{~nm}$ (Fig. 3b), i.e. well below even the size of native unmodified glycogen $(53.4 \pm 0.2 \mathrm{~nm})$; even the nanoparticle core must therefore shrink upon coil-to-globule transition and must be POX-modified. There is also visible phase separation and aggregation into a nanostructured material above the CPT (see Fig. 3).

We measured CPT as a function of the concentration in solution to obtain a complete phase diagram for the polymer solution (Fig. 4).

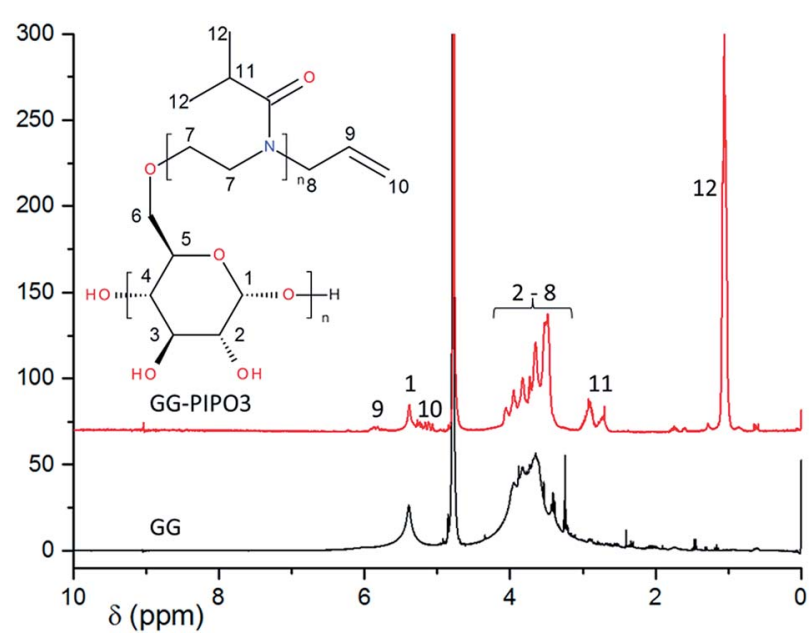

Fig. $2{ }^{1} \mathrm{H}$ NMR spectra of glycogen and of the glycogen-graft-poly(2isopropyl-2-oxazoline) GG-PIPO3.

Table 1 The theoretical and experimentally found compositions of the graft copolymers; $M_{n, \text { graft }}-$ molecular weight of the POX grafts, $W_{\mathrm{POX}}-$ weight fraction of POX in the hybrid copolymer

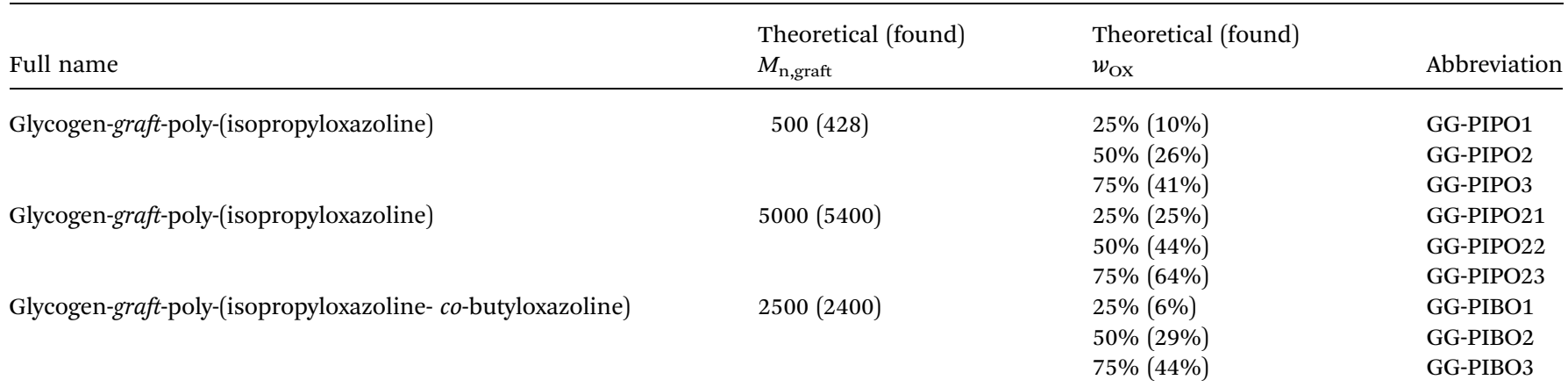




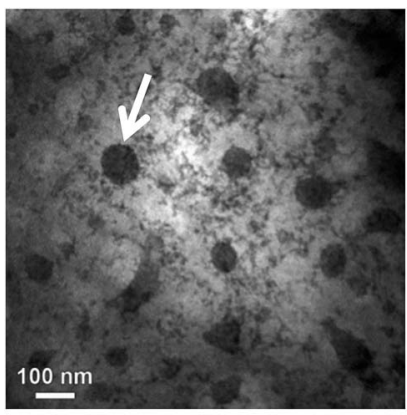

$\mathbf{a}$

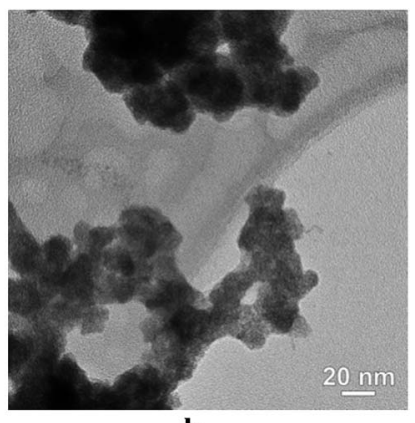

b
Fig. 3 TEM image of a solution of polymer GG-PIBO3 in $0.9 \%$ aqueous $\mathrm{NaCl}$ at $20^{\circ} \mathrm{C}\left((\mathrm{a})\right.$, below LCST) and at $37^{\circ} \mathrm{C}((\mathrm{b})$, body temperature). The small diffuse entities visible across the background are uranium salt particles.

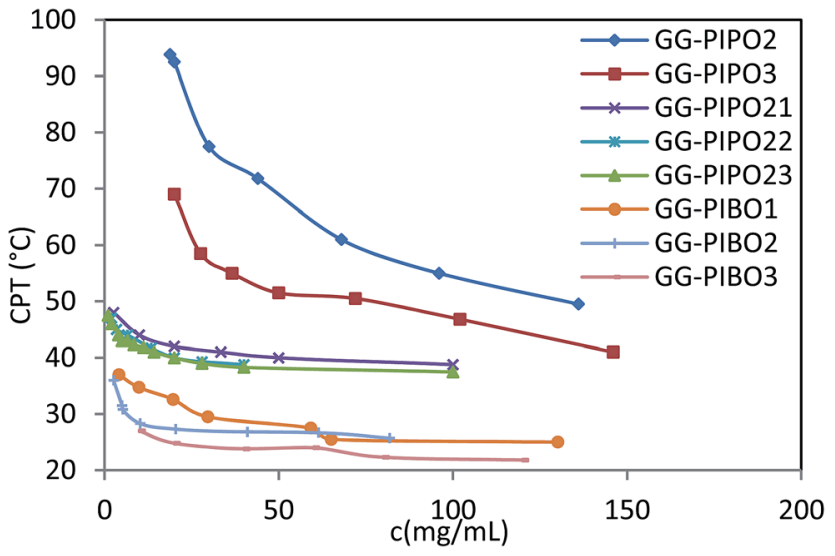

Fig. 4 Cloud point temperature (CPT) of the thermoresponsive GGgraft-polyoxazoline solutions in $0.9 \% \mathrm{NaCl}$ as a function of concentration (c). Results for GG-PIPO1 are not displayed (as the CPT was not observed between 25 and $90^{\circ} \mathrm{C}$ for polymer concentrations of $10-$ $100 \mathrm{mg} \mathrm{mL}^{-1}$ ).

As expected, the CPT of all of the glycogen-graft-poly(2-alkyl2-oxazolines) (Fig. 4) rises with decreasing concentration, content of polyoxazoline, molar mass of the polyoxazoline graft and hydrophobicity of the alkyl groups.

The most significant influence on the CPT is from the graft length. For the shorter grafts $\left[M_{\mathrm{n} \text {,theoretical }}=500\right.$, i.e. $4.42-\mathrm{mer}$ for poly(2-isopropyl-2-oxazoline)], increasing the content of polymer graft significantly decreases the CPT (GG-PIPO1 (where CPT $>90{ }^{\circ} \mathrm{C}$ for any concentration) $>$ GG-PIPO2 $>$ GG-PIPO3). There is also a strong concentration dependence of the CPT for the graft copolymers with shorter grafts (the GG-PIPO polymers), which is strongest for the polymer with a lower POX content (GG-PIPO2). This is consistent with our previous observation $^{20}$ of multiblock poly(2-oxazolines), that for shorter chains, the total hydrophobicity of the molecule plays a more significant role than the cloud point temperature of POX itself, and the possibility of the POX chains getting close to each other (concentration dependence, content of POX in polymer) is obviously a key factor. This is also supported by the fact that for longer grafts $\left[M_{\mathrm{n}, \text { theoretical }}=5000\right.$, i.e. 44.2-mer for poly(2- isopropyl-2-oxazoline)] both the effects of the polymer concentration in solution and the POX content in the graft copolymer are very low, much smaller than in the case of shorter grafts.

Copolymerization with the more hydrophobic comonomer 2butyl-2-oxazoline (GG-PIBO1, GG-PIBO2 and GG-PIBO3) shifts the CPT to lower temperatures, which is consist with other poly(2-isopropyl-2-oxazoline) systems, to the desired region between room and body temperature that is useful for further biomedicinal applications. The trends in the concentration and POX content dependences of the CPT however remain the same for the POX copolymer graft polymer as for the poly(2-isopropyl2-oxazoline) graft polymer. The 2400 Da grafts in the GG-PIBO polymers seem to be more similar in their influence on the CPT concentration dependence trends to the 5400 grafts in the GG-PIPO polymers than to the 428 Da grafts in the GG-PIPO polymers.

The solution properties of the solutions of glycogen conjugates in water were determined by DLS. Intensity weighted distribution functions for the solution of the G-PIPO21 conjugate (Fig. 5) show that the conjugate, with a $R_{\mathrm{h}}$ of $30 \mathrm{~nm}$ at $25^{\circ} \mathrm{C}$, has thermosensitive properties. The PIPO21 is shrinking with increasing temperature and this is manifested as a decrease in $R_{\mathrm{h}}$, consistent with the cryo-TEM images (Fig. 3a and b). The decrease in the $R_{\mathrm{h}}$ values occurs for two reasons. The first reason is a coil-to-globule transition of the PIPO chains on the glycogen surface. The second reason is a finite concentration of the solutions. At $c=1.0 \mathrm{wt} \%$, DLS measurements provide an apparent value of $R_{\mathrm{h}}$, which is controlled by a $k_{\mathrm{D}}$ parameter - the concentration diffusion coefficient. It was shown ${ }^{23}$ that in dilute solutions, the $k_{\mathrm{D}}$ parameter is related to the second viral coefficient $A_{2}$ and molecular weight $M_{\mathrm{w}}$ by the equation

$$
k_{\mathrm{D}}=2 A_{2} M_{\mathrm{w}}-k_{\mathrm{f}}-\bar{\nu}
$$

here $k_{\mathrm{f}}$ and $\bar{\nu}$ are the concentration friction coefficient and partial specific volume, respectively. In contrast to $A_{2}, k_{\mathrm{f}}$ is always positive. Therefore, in general, the case sign of $k_{\mathrm{D}}$ is determined by all three parameters $-M_{\mathrm{w}}, A_{2}$ and $k_{\mathrm{f}}$ (the partial

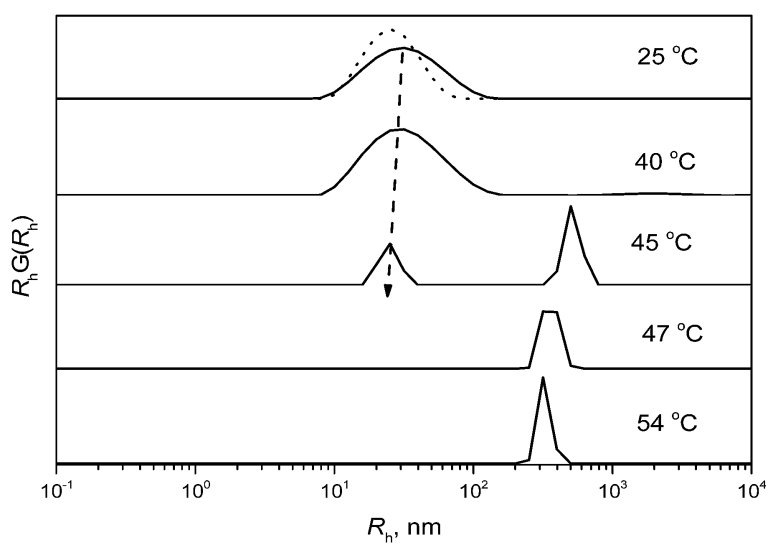

Fig. 5 The intensity weighted distribution function of the nanoparticles, obtained from DLS data for GG-PIPO21 at different temperatures; $\theta=90^{\circ}$; polymer concentration $(c)=1.0 \mathrm{wt} \%$. The dotted line represents unmodified glycogen. 
specific volume value can be neglected). At $45{ }^{\circ} \mathrm{C}$, a new peak with a much higher $R_{\mathrm{h}}$ value starts to be dominant in the intensity weighted distribution function, implying the existence of large aggregates in solution, which corresponds to a phaseseparated polymer.

To find the precise position of the CPT for each concentration, the dependence of the $R_{\mathrm{h}}$ and intensity was monitored as a function of temperature (Fig. 6 and 7). For polymers GG-PIPO1, GG-PIPO2 and GG-PIPO3, no divergence was observed as a function of temperature (Fig. 6).

The lack of a cloud point temperature in the $R_{\mathrm{h}}$ temperature dependence results of GG-PIPO1, GG-PIPO2, and GG-PIPO3 (Fig. 6) results from a low concentration of the polymer solution (however, at higher concentrations a CPT exists, except for the most hydrophilic species PIPO1 (see Fig. 4)). In these cases the CPT is expected to be above $95{ }^{\circ} \mathrm{C}$.

In contrast, for GG-PIPO21, GG-PIPO22 and GG-PIPO23, both the $R_{\mathrm{h}}$ and the intensity show divergence at a specific point that corresponds to the CPT (Fig. 7). It can be observed that with increasing temperature, large aggregates occur in the solution above the CPT. Such aggregates were witnessed by TEM, where entities with a size above $100 \mathrm{~nm}$ were present in the solution (Fig. 3B).

Next, the detailed structures of the modified and nonmodified glycogens were investigated by SAXS. SAXS techniques have served as a powerful tool for analysing nanoparticle structures and suspensions of both concentrated and dilute polymer solutions. ${ }^{24-26}$

All samples were measured at three temperatures: $25^{\circ} \mathrm{C}, 35$ ${ }^{\circ} \mathrm{C}$, and $45^{\circ} \mathrm{C}$ at a concentration of $1.0 \mathrm{wt} \%$ (Fig. 8A and $\mathrm{B}$ and Table 2). Predictably, no changes were observed for the unmodified glycogen with increasing temperature. The scattering profiles of the solutions of glycogen conjugates GGPIPO1, GG-PIPO2, and GG-PIPO3 also show no temperature sensitivity at $c=1.0 \%$, which is in agreement with DLS data (Fig. 8).

Since glycogen has a hyperbranched structure, a Dozier mode ${ }^{27}$ was used to fit the scattering data of the oyster glycogen and its conjugates (Fig. 9).

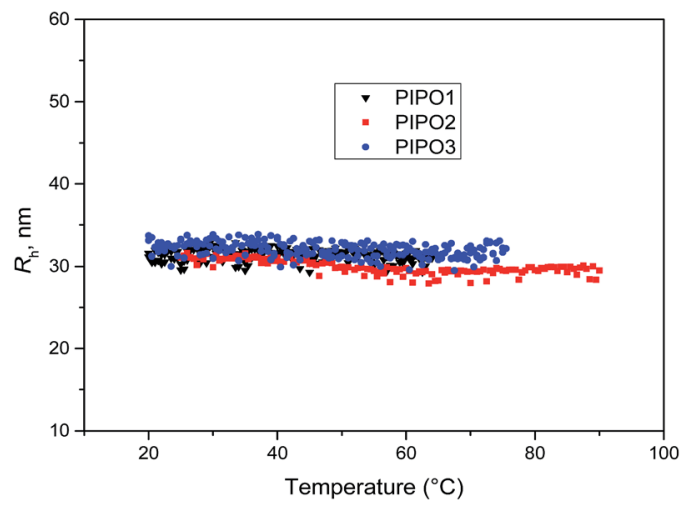

Fig. 6 The temperature dependence of the $R_{\mathrm{h}}$ values obtained from the intensity weighted distribution functions for GG-PIPO1, GGPIPO2, and GG-PIPO3; polymer concentration (c) = 1.0 wt $\%$.

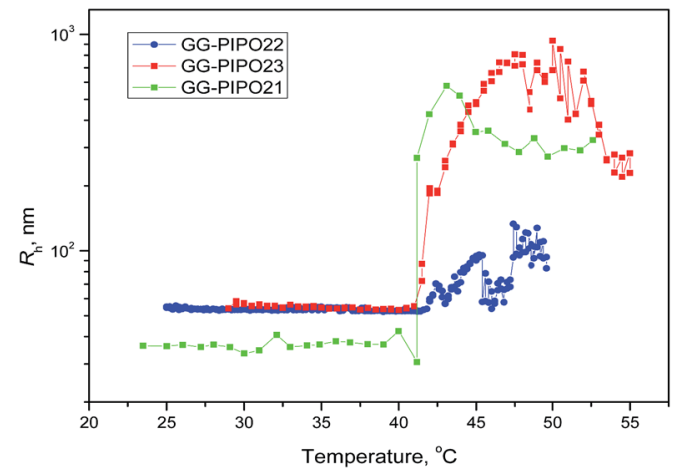

Fig. 7 The temperature dependence of the $R_{\mathrm{h}}$ values obtained from intensity weighted distribution functions for GG-PIPO21, GG-PIPO22, and GG-PIPO23; polymer concentration (c) $=1.0 \mathrm{wt} \%$.
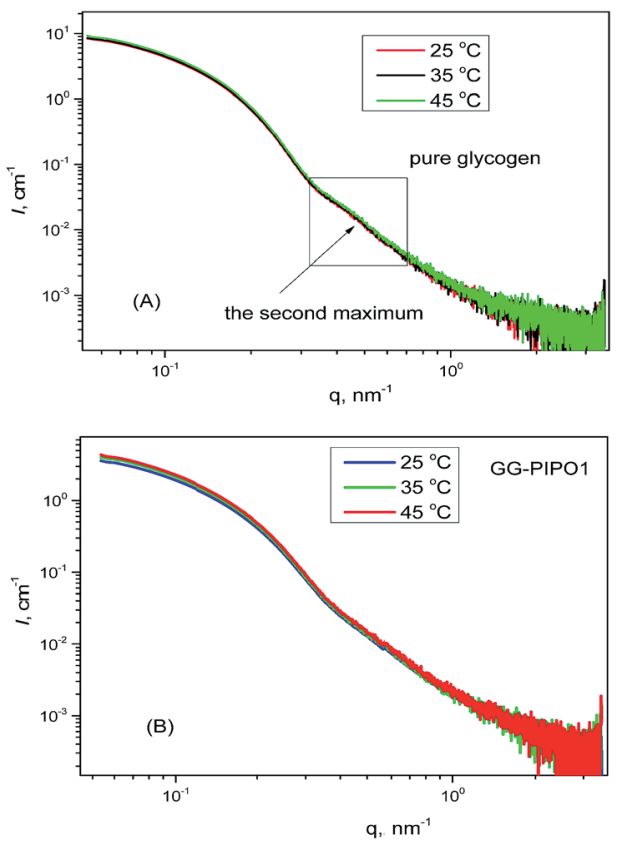

Fig. 8 SAXS curves for solutions of non-modified glycogen (A) and GG-PIPO1 (B), taken at $T=25,35$, and $45^{\circ} \mathrm{C}$; $c=1.0 \mathrm{wt} \%$.

For a star polymer Dozier developed a scattering function:

$$
\begin{aligned}
I_{\text {DozierStar }}\left(q, I_{0}, R_{\mathrm{g}}, \alpha, \nu, \varepsilon\right)= & I_{0} \exp \left(-\frac{q^{2} R_{\mathrm{g}}{ }^{2}}{3}\right) \\
& +\frac{4 \pi \alpha}{q \varepsilon} \Gamma(\mu) \frac{\sin (\mu \arctan (q \varepsilon))}{\left(1+q^{2} \varepsilon^{2}\right)^{\mu / 2}}
\end{aligned}
$$

This model fitting provided the gyration radius $\left(R_{\mathrm{g}}\right)$, excluded volume parameter $(\nu)$, and scattered intensity extrapolated to zero $q$ (Table 2). The excluded volume parameter $\nu$ varies in the range $0.33-1.0$. The value of $\nu$ of 0.5 stands for the theta-solvent. $\alpha$ in this model is a scale parameter for the fractal term; $\varepsilon$ is an exponential damping length in the mass fractal.

SAXS experiments indicate the conformation changes of the conjugates due to modification (Fig. 10, Table 1). Non-modified 
Table 2 The fitting parameters from SAXS at $25^{\circ} \mathrm{C}\left(45^{\circ} \mathrm{C}\right)^{a}$

\begin{tabular}{|c|c|c|c|c|c|c|c|}
\hline Sample & Oyster GG & GG-PIPO1 & GG-PIPO2 & GG-PIPO3 & GG-PIPO21 & GG-PIPO22 & GG-PIPO23 \\
\hline$R_{\mathrm{g}}, \mathrm{nm}$ & $13.6(13.6)$ & $11.9(11.9)$ & $12.0(12.0)$ & $12.2(12.1)$ & $11.7(11.7)$ & $12.3(12.3)$ & $12.7(12.7)$ \\
\hline$\alpha$ & $0.48(0.52)$ & $0.27(0.33)$ & $0.33(0.39)$ & $0.22(0.25)$ & $0.24(0.28)$ & $0.79(0.83)$ & $0.31(0.93)$ \\
\hline$\nu$ & $0.36(0.36)$ & $0.39(0.39)$ & $0.39(0.39)$ & $0.39(0.38)$ & $0.37(0.38)$ & $0.37(0.37)$ & $0.41(0.37)$ \\
\hline
\end{tabular}

${ }^{a}$ The bold values show the growth of the glycogen with modification.

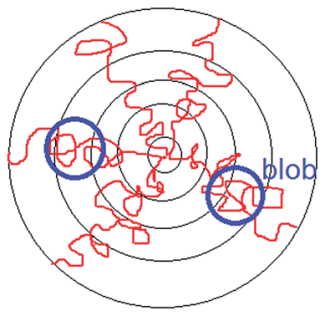

Fig. 9 The structure of a Dozier star polymer.
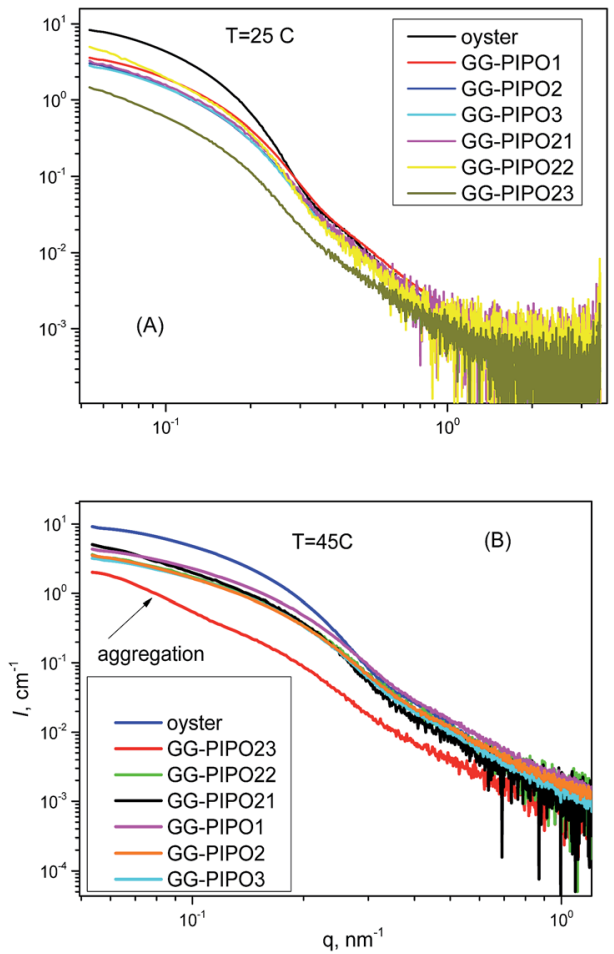

Fig. 10 SAXS curves for the solutions of glycogen and glycogen conjugates at $T=25^{\circ} \mathrm{C}(\mathrm{A})$ and $T=45^{\circ} \mathrm{C}(\mathrm{B}) ; \mathrm{c}=1.0 \mathrm{wt} \%$.

glycogen has the lowest polydispersity as could be witnessed by the presence of the second maximum on the scattering curve (Fig. 8A and B and Fig. 11). Another feature could be also noted: a substantial decrease with modification in the scattered intensity extrapolated to zero $q$ (Fig. 10). We can assume that chemical modification distorts the spherical structure of nonmodified glycogen. The $R_{\mathrm{g}}$ value increases with increasing grafting density in the series GG-PIPO1-GG-PIPO3 and GG-
PIPO21-GG-PIPO23 (Table 2). This proves the successfulness of the modification. The exponential damping length value shows that the most significant changes with temperature occur for the GG-PIPO23 conjugate. For GG-PIPO23 (Fig. 11), the presence of large particles was also visible at a low $q$ range. Such findings indicate the onset of aggregation at temperatures near the CPT. To conclude, the SAXS data support the successful chemical modification of glycogen. The size of the glycogen grows with an increase in the length and grafting density of the polyoxazoline. Nevertheless, only the sample with the highest PIPO grafting density and graft length (GG-PIPO23) revealed thermosensitivity, since the SAXS experiments were conducted within a smaller temperature range than for the the DLS.

In the ITC experiment, a BSA solution was titrated into PBS buffer (Fig. 12). All integrated curves have the same behavior which shows that there are no interactions between BSA and the glycogens (the drop at molar ratio 2 is due to the heat of dilution of BSA).

Non-interaction with blood proteins is one of the prerequisites of biocompatibility for polymers to be used in solution, so we can expect a biocompatibility of the studied hybrid copolymers in this case.

In our previous work, we studied different thermoresponsive nanoparticle-forming poly[2-methyl-2-oxazoline-block-(2-isopropyl-2-oxazoline-co-2-butyl-2-oxazoline)-block-2-methyl-2-oxazoline $]^{20}$ ABA triblock copolymers as well as Pluronic F127 surfactant-stabilized poly(2-isopropyl-2-oxazoline-co-2-butyl-2oxazoline) nanoparticles and free poly(2-isopropyl-2-oxazolineco-2-butyl-2-oxazoline). ${ }^{28}$ These are systems with the same

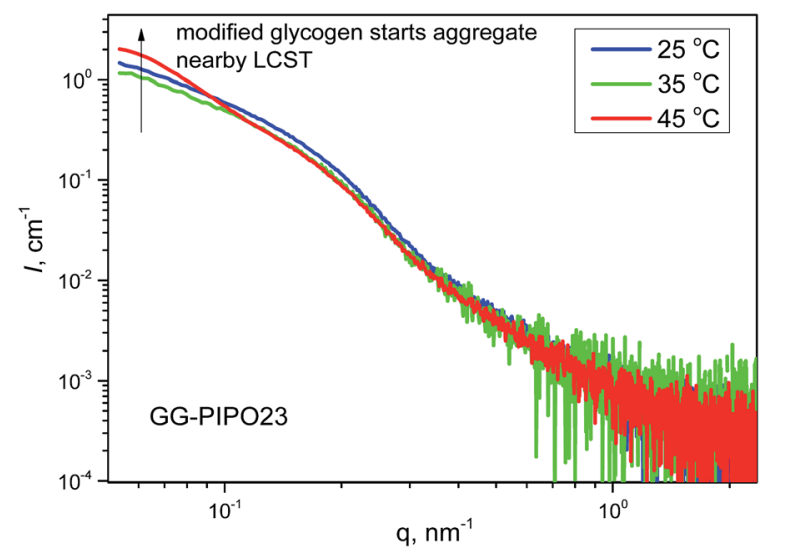

Fig. 11 SAXS curves for solutions of GG-PIPO23, taken at different temperatures; $c=1.0 \mathrm{wt} \%$. The solid lines are fitted lines. 


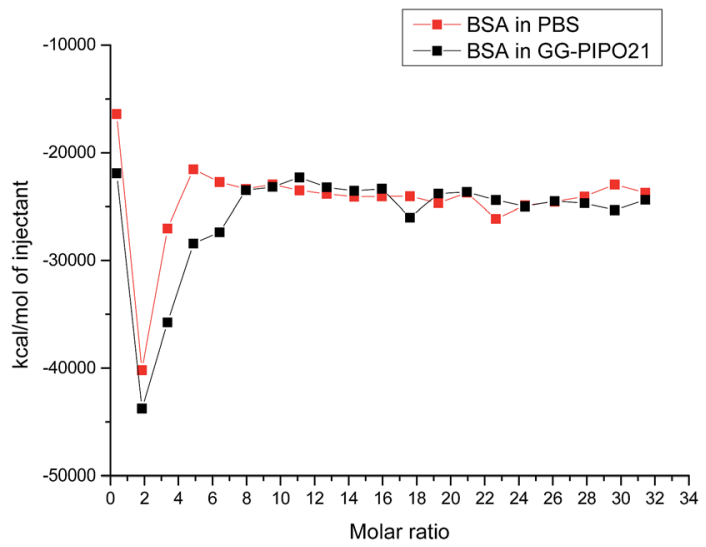

Fig. 12 Isothermal titration calorimetry of PBS (blank) and a GGPIPO22 solution in PBS.

thermoresponsive part but a different hydrophilic part and general polymer architecture. When compared to the system described in this paper, glycogen has less influence on the overall thermoresponsive behavior of the thermoresponsive grafts than the poly(2-methyl-2-oxazoline) block in the ABA triblock copolymers has if the thermoresponsive grafts are at least $2.5 \mathrm{kDa}$ long. In this case, such a system is closer in its temperature-dependent solution behavior to the surfactantstabilized nanoparticles or to free poly(2-isopropyl-2-oxazolineco-2-butyl-2-oxazoline) than to the ABA triblock copolymers. On the other hand, for the shorter $500 \mathrm{Da}$ grafts, the overall hydrophobicity/hydrophilicity of the whole system including glycogen plays a crucial role, as for the ABA triblock copolymers.

\section{Conclusions}

We describe a new platform of hybrid graft copolymers, glycogen-graft-thermoresponsive polyoxazoline. These polymers are easy-to-prepare and relatively inexpensive. The properties of these compounds can be easily modified by changes in the composition, molar mass and the content of polyoxazoline grafts. It was proven that all of the synthesized glycogen conjugates, except for the most hydrophilic conjugate PIPO1, possess a cloud point temperature. By simple variation of the polyoxazoline molecular weight, the solution properties including the LCST value could be easily tuned in a broad temperature range providing an ideal tool for the creation of thermosensitive polymers. The structure of the glycogen conjugate was described using a Dozier star model. The $R_{\mathrm{g}}$ value increases with increasing of the grafting density for the series GG-PIPO1-GG-PIPO3 and GG-PIPO21-GG-PIPO23, which proves the successfulness of the grafting.

\section{Conflict of interest}

The authors declare no competing financial interests.

\section{Acknowledgements}

We gratefully acknowledge financial support from the Academy of Sciences of the Czech Republic (grant \# M200501201), the Grant Agency of the Czech Republic (grant \# 13-08336S), the Ministry of Education, Youth and Sports of the Czech Republic (grant \# MSMT KONTAKT II LH14079) and the Joint Project ASCR/CONICET no. 2012CZ006.

\section{Notes and references}

1 T. A. Tirone and F. C. Brunicardi, World J. Surg., 2001, 25, 461-467.

2 J. Shearer and T. E. Graham, Can. J. Appl. Physiol., 2002, 27, 179-203.

3 B. W. N. A. Campbell and R. J. Heyden, Biology: Exploring Life, Pearson Prentice Hall, Boston, 1st edn, 2006.

4 D. J. Manners, Carbohydr. Polym., 1991, 16, 37-82.

5 K. K. Rybicka, Tissue Cell, 1996, 28, 253-265.

6 S. K. Filippov, O. Sedlacek, A. Bogomolova, M. Vetrik, D. Jirak, J. Kovar, J. Kucka, S. Bals, S. Turner, P. Stepanek and M. Hruby, Macromol. Biosci., 2012, 12, 1731-1738.

7 R. Hoogenboom and H. Schlaad, Polymers, 2011, 3, 467-488.

8 R. Luxenhofer, Y. C. Han, A. Schulz, J. Tong, Z. J. He, A. V. Kabanov and R. Jordan, Macromol. Rapid Commun., 2012, 33, 1613-1631.

9 A. Popelka, J. Kronek, I. Novak, A. Kleinova, M. Micusik, M. Spirkova and M. Omastova, Vacuum, 2014, 100, 53-56.

10 L. T. T. Trinh, H. M. L. Lambermont-Thijs, U. S. Schubert, R. Hoogenboom and A. L. Kjoniksen, Macromolecules, 2012, 45, 4337-4345.

11 O. Sedlacek, B. D. Monnery, S. K. Filippov, R. Hoogenboom and M. Hruby, Macromol. Rapid Commun., 2012, 33, 16481662.

12 R. Andre, M. N. Tahir, F. Natalio and W. Tremel, FEBS J., 2012, 279, 1737-1749.

13 K. Aoi, A. Takasu, M. Okada and T. Imae, Macromol. Chem. Phys., 1999, 200, 1112-1120.

14 R. Nakamura, K. Aoi and M. Okada, Macromol. Biosci., 2004, 4, 610-615.

15 J. Cheng, M. Park and J. Hyun, Cellulose, 2014, 21, 16991708.

16 M. A. Karaaslan, G. Z. Gao and J. F. Kadla, Cellulose, 2013, 20, 2655-2665.

17 H. Madry, A. Rey-Rico, J. K. Venkatesan, B. Johnstone and M. Cucchiarini, Tissue Eng., Part B, 2014, 20, 106-125.

18 M. Hruby, P. Pouckova, M. Zadinova, J. Kucka and O. Lebeda, Eur. J. Pharm. Sci., 2011, 42, 484-488.

19 C. Diab, Y. Akiyama, K. Kataoka and F. M. Winnik, Macromolecules, 2004, 37, 2556-2562.

20 M. Hruby, S. K. Filippov, J. Panek, M. Novakova, H. Mackova, J. Kucka, D. Vetvicka and K. Ulbrich, Macromol. Biosci., 2010, 10, 916-924.

21 J. Jakes, Czech. J. Phys., 1988, 38, 1305-1316.

22 P. Stepanek, in Dynamic Light Scattering: The Method and Some Applications, ed. W. Brown, Clarendon Press, Oxford, 1993, pp. 175-241. 
23 H. Yamakawa, Modern Theory of Polymer Solutions, Harper and Row Publishers, New York, 1971.

24 D. Gromadzki, S. Filippov, M. Netopilik, R. Makuska, A. Jigounov, J. Plestil, J. Horsky and P. Stepanek, Eur. Polym. J., 2009, 45, 1748-1758.

25 B. Angelov, A. Angelova, S. K. Filippov, T. Narayanan, M. Drechsler, P. Stepanek, P. Couvreur and S. Lesieur, J. Phys. Chem. Lett., 2013, 4, 1959-1964.
26 S. K. Filippov, P. Chytil, P. V. Konarev, M. Dyakonova, C. M. Papadakis, A. Zhigunov, J. Plestil, P. Stepanek, T. Etrych, K. Ulbrich and D. I. Svergun, Biomacromolecules, 2012, 13, 2594-2604.

27 W. D. Dozier, J. S. Huang and L. J. Fetters, Macromolecules, 1991, 24, 2810-2814.

28 J. Panek, S. K. Filippov, M. Hruby, M. Rabyk, A. Bogomolova, J. Kucka and P. Stepanek, Macromol. Rapid Commun., 2012, 33, 1683-1689. 